\title{
Patients' understanding of health information in Germany
}

This article was published in the following Dove Press journal:

Patient Preference and Adherence

\author{
Florian Tille ${ }^{1,2}$ \\ Heide Weishaar ${ }^{3}$ \\ Bernhard Gibis ${ }^{2}$ \\ Susanne Schnitzer' \\ 'Charité - Universitätsmedizin Berlin, \\ Berlin 10117, Germany; ${ }^{2}$ Kassenärztliche \\ Bundesvereinigung, Berlin 10623, \\ Germany; ${ }^{3}$ Hertie School of Governance, \\ Berlin 10117, Germany
}

Background: Patient-physician communication and textual health information are central to health care. Yet, how well patients understand their physicians and written materials is under-studied.

Objectives: Focusing on outpatient health care in Germany, the aim of this research was to assess patients' levels of understanding oral and written health information and to identify associations with socioeconomic variables.

Methods: This analysis drew on a 2017 health survey ( $n=6,105$ adults 18 years of age and above). Measures for the quality of patient-physician communication were derived from the Ask Me 3 program questions for consultations with general practitioners (GPs) and specialists (SPs), and for textual health information via a question on the comprehensibility of written materials. Correlations with socioeconomic variables were explored using bivariate and multivariable logistic regression analyses.

Results: Over $90 \%$ of all respondents reported that they had understood the GP's and SP's explanations. A lack of understanding was most notably correlated with patients' selfreported very poor health (odds ratio [OR]: 5.19; 95\% confidence interval [CI]: 2.23-12.10), current health problem (OR: 6.54, CI: 1.70-25.12) and older age (65 years and above, OR: 2.97 , CI: $1.10-8.00)$. Fewer patients reported that they understood written materials well ( $86.7 \%$ for last visit at GP, $89.7 \%$ for last visit at SP). Difficulties in understanding written materials were strongly correlated with basic education (OR: 4.20, CI: 2.76-6.39) and older age (65 years and above, OR: 2.66, CI: 1.43-4.96).

Conclusions: In order to increase patients' understanding of health information and reduce inequalities among patient subgroups, meeting the communication needs of patients of older age, low educational status and with poor health is essential.

Keywords: patient-physician communication, written health information, Ask Me 3, health literacy, socioeconomic variables, health survey

\section{Introduction}

The concept of health literacy (HL) has undergone considerable change since it emerged in the 1990s- ${ }^{1-3}$ While early definitions framed HL as the individual's reading and writing abilities to functionally handle information on health problems, ${ }^{2,4,5}$ the conceptualization evolved to include interactive and critical skills, conceiving HL as a personal and collective resource for health-promoting behavior in different settings. ${ }^{4,6}$ Sørensen et al define HL as "people's knowledge, motivation and competences to access, understand, appraise and apply health information in order (...) to maintain or improve quality of life (...)". The World Health Organization and others consider it a determinant, mediator and moderator of
Correspondence: Florian Tille

Charité - Universitätsmedizin Berlin,

Virchowweg 22, Berlin 10117, Germany

Tel +491 7656594125

Email florian.tille@charite.de 
health. ${ }^{2,6,8,9}$ Yet, empirical studies show that levels of HL are alarmingly low across populations, with up to half of all adults demonstrating insufficient HL competences in North American and European surveys ${ }^{13}$ Also, HL levels are unequally distributed- ${ }^{15}$ making $\mathrm{HL}$ a potential factor for increasing health inequalities ${ }^{14,16,17}$ and hence a pivotal public health issue. ${ }^{15}$

While existing findings underline the need to raise levels of HL, answers to the question of how this might be done remain ambiguous. ${ }^{17}$ Approaches that consider HL to be a personal set of skills and abilities modifiable through individual learning ${ }^{18}$ have been expanded by those that draw attention to the complexity of the health systems that people interact with. ${ }^{18,19}$ Proponents of organizational $\mathrm{HL}^{3,18,20}$ highlight that an individual's ability to adequately deal with health information substantially depends on structural factors, for example on how challenging it is to access, understand, appraise and apply referenced, actionable health information. ${ }^{4,18,20}$ As a result, agreement exists that in order to increase HL, both individual resources need to be enhanced and the structural environment, decision processes and systems have to be adapted. ${ }^{2,8}$ It is also acknowledged that those who design, disseminate and communicate health information play a crucial role for people's HL as they can improve the comprehensibility of information, thereby enabling the recipients to make informed decisions. The critical contribution that health professionals can make by providing adequate and tailored communication has thus been highlighted as a major aspect of strategies for raising HL levels among patients. ${ }^{3,21}$

Previous research has focused on deficits in the communication between health professionals - especially physicians - and health system users, leading to calls for action to enhance communication competences of health professionals. ${ }^{13,17,22}$ However, while recent data on population HL levels are available for Germany, ${ }^{13}$ to date no research has examined patients' assessment of the communication skills of health professionals and of the health information they receive.

This study addresses this research gap by investigating whether patients who receive outpatient health care in Germany understand the physicians' - general practitioners (GPs) commonly constitute the first, specialists (SPs) the second point of contact in health care in Germany - explanations during the medical consultation. To account for textual communication, we also analyzed how well these patients understand written health information. In addition to overall levels of understanding and in order to identify potential predictors of health inequalities, differences between sub-populations were explored by analyzing the associations between reported levels of understanding and socioeconomic variables.

This research contributes to a better understanding of HL in Germany by determining to what extent oral patient-physician communication and written health materials in outpatient care may be considered building blocks to be addressed when developing strategies to reduce systemic barriers to processing health information.

\section{Data and methods}

Our analyses are based on household data collected via a 2017 cross-sectional national survey of health service users (Versichertenbefragung). The survey was conducted by the German National Association of Statutory Health Insurance Physicians (Kassenärztliche Bundesvereinigung [KBV]), in cooperation with the Institute of Medical Sociology and Rehabilitation Science of Charité University and the research institute Forschungsgruppe Wahlen (FGW). ${ }^{23}$

\section{Study population and sampling design}

The survey sample was derived from all German-speaking adults living in a household with a landline phone connection in Germany. A random sample was generated through regional stratification of the population, selection of landline phone numbers via randomized last digit dialing and selection of the respondent through the last birthday method. ${ }^{23}$ Computer-assisted telephone interviews (CATI) were conducted in German language by the FGW between 15 May and 27 June 2017. The data were weighted for the number of landlines and persons per household, as well as for gender, age and education according to their nominal distribution across the adult population in Germany. ${ }^{24}$ The total sample comprised 6,105 individuals.

\section{Study instrument and variables}

The survey questionnaire was drafted by an expert group of the KBV, Charité and FGW. It included three questions on patient-physician communication and one on understanding of written health information. The questions on patient-physician communication were derived from the US Institute for Healthcare Improvement's Ask Me 3 program (AM3), which encourages patients to specifically ask their physician three questions during the medical consultation: "What is my main 
health problem?", "What do I need to do?" and "Why is it important for me to do this?". The responses to these questions are supposed to enhance patients' understanding of their own health condition and treatment options as well as to improve adherence to the physician's recommendations ${ }^{27}$ Participants who reported that during their last consultation in the previous twelve months the physician had answered these questions were asked if they had understood the corresponding explanations. The answers were measured on a dichotomous scale as "yes" or "no". Respondents were further asked how well they generally understood written materials on health, with answer options being "very well", "well", "not well" and "not well at all" (for translation of the questions, see Table 1). These questions were treated as dependent variables in the analyses.

Age and gender as well as education, occupational status, type of health insurance (statutory or private) and nationality were included as variables for socioeconomic variables in our analyses. Since studies have shown associations between health-related socioeconomic variables and HL, ${ }^{11,12,28}$ self-reported health, suffering from chronic disease and reason for last physician consultation were added to the models (see Table 2).

\section{Statistical analyses}

Total percentages of the four questions, differentiated by GPs and SPs and stratified by socioeconomic variables, were calculated. The results were tested for statistical significance between the subgroups of the GP and SP samples. Corresponding to the different measurement scales, chi-square tests were applied to the answers on patient-physician communication and Kruskal-Wallis and Mann-Whitney $U$ tests to those on written materials.

Table I Translation of questions on patient-physician communication and on written health information in the 2017 national survey of health service users

\section{Translated question}

Did your physician's explanations help you to better understand what your main health problem is?*

Did your physician's explanations help you to better understand what exactly you could do about this health problem?*

Did your physician's explanations help you to better understand why you should do this?*

When you read something on health, do you generally understand this very well, well, not well, or not well at all?

Note: *Respondents were informed that they were to provide their subjective assessment of their experience based on their last physician's visit in the previous 12 months. Answer categories: "yes", "no".
For the multivariable analysis, binary logistic regression was performed to identify characteristics that were associated with negative responses while controlling for confounding factors. In order to do this, the answers for the question on written materials were dichotomized into "well" (combining responses "very well" and "well") and "not well" (combining "not well" and "not well at all"). The regression models were estimated and recalculated iteratively through backward selection to remove nonsignificant independent variables. ${ }^{23}$ All results were adjusted for age and gender in the multivariable analyses.

SPSS Statistics version 23 was used for the calculations. Missing values were deleted listwise and the significance level was determined at 5\%. The multivariable results are depicted as odds ratios (OR) with 95\% confidence intervals (CI).

\section{Results}

Of the total sample of 6,105 respondents, 5,158 people reported at least one GP or SP consultation within the previous twelve months. From this sub-sample, two weighted samples, one consisting of 3,056 respondents who last visited a GP and one of 2,102 who last visited an SP, were derived and used for the analyses (Table 2).

The majority of all surveyed patients reported that the physician's explanations during the last consultation had helped them to understand what their main health problem was (94.0\% for last visit at GP, 93.0\% for last visit at SP), what they could do about it (95.3\% GP, 95.8\% SP) and why they should do this (98.9\% GP, 97.5\% SP) (Tables 3 and 4). Fewer patients reported to generally understand written health materials very well or well, namely $86.7 \%$ of those who last consulted a GP and $89.7 \%$ of those who last consulted an SP (Tables 3 and 4).

\section{Communication at the GP}

Self-reported health was bivariately associated with whether patients had understood their main health problem during the last GP consultation: Understanding decreased with worse self-reported health $(95.5 \%$ of patients in excellent/very good health vs $87.7 \%$ of patients in very poor health) (Table 3). Also, patients who consulted the GP for a current health problem reported that they had understood the explanations on what to do about their health problem statistically significantly less often than those who visited the GP for other reasons $(94.5 \%$ vs $99.0 \%$ due to a preventive examination) (Table 3 ). 
Table 2 Description of the sample statistics ( $\mathrm{N}$ for "Total" =6, 105; $\mathrm{n}$ for "Last visit at GP" =3,056; $\mathrm{n}$ for "Last visit at SP" =2, 102 ; weighted)

\begin{tabular}{|c|c|c|c|c|c|c|}
\hline & \multicolumn{2}{|l|}{ Total } & \multicolumn{2}{|c|}{ Last visit at GP } & \multicolumn{2}{|c|}{ Last visit at SP } \\
\hline & Quantity & Percentage* & Quantity & Percentage* & Quantity & Percentage* \\
\hline \multicolumn{7}{|l|}{ Age } \\
\hline 18 to 34 years & $\mathrm{I}, \mathrm{I} 78$ & 19.3 & 568 & 18.6 & 400 & 19.0 \\
\hline 35 to 49 years & $\mathrm{I}, 477$ & 24.2 & 676 & 22.1 & 519 & 24.7 \\
\hline 50 to 64 years & 1,637 & 26.8 & 792 & 25.9 & 604 & 28.7 \\
\hline 65 years and above & 1,812 & 29.7 & 1,020 & 33.4 & 579 & 27.6 \\
\hline \multicolumn{7}{|l|}{ Gender } \\
\hline Female & 3,230 & 52.9 & 1,626 & 53.2 & 1,206 & 57.4 \\
\hline Male & 2,875 & 47.1 & 1,430 & 46.8 & 896 & 42.6 \\
\hline \multicolumn{7}{|l|}{ Educational status } \\
\hline Higher education & 1,974 & 32.8 & 892 & 29.6 & 779 & 37.5 \\
\hline Intermediate education & 1,969 & 32.7 & 961 & 31.9 & 687 & 33.1 \\
\hline Basic education & 1,952 & 32.4 & 1,095 & 36.3 & 577 & 27.8 \\
\hline No degree & 86 & 1.4 & 40 & 1.3 & 27 & 1.3 \\
\hline Still at school & 40 & 0.7 & 27 & 0.9 & 7 & 0.3 \\
\hline \multicolumn{7}{|l|}{ Occupational status } \\
\hline Full-time & 2,395 & 39.8 & 1,128 & 37.5 & 780 & 37.6 \\
\hline Part-time/temporary & 898 & 14.9 & 460 & 15.3 & 318 & 15.3 \\
\hline Unemployed & 106 & 1.8 & 54 & 1.8 & 36 & 1.8 \\
\hline Retired & 1,999 & 33.2 & 1,104 & 36.7 & 675 & 32.6 \\
\hline Training/volunteering/school & 272 & 4.5 & 129 & 4.3 & 105 & 5.1 \\
\hline Parental leave/not-employed & 348 & 5.8 & 135 & 4.5 & 157 & 7.6 \\
\hline \multicolumn{7}{|l|}{ Type of health insurance } \\
\hline Private & 772 & 12.8 & 291 & 9.6 & 327 & 15.7 \\
\hline Statutory & 5,266 & 87.2 & 2,732 & 90.4 & $\mathrm{I}, 755$ & 84.3 \\
\hline \multicolumn{7}{|l|}{ Self-reported health status } \\
\hline Excellent/very good & 2,202 & 36.4 & 1,000 & 33.0 & 693 & 33.4 \\
\hline Good & 2,607 & 43.1 & 1,395 & 46.1 & 865 & 41.6 \\
\hline Poor & 959 & 15.9 & 487 & 16.1 & 411 & 19.8 \\
\hline Very poor & $28 I$ & 4.6 & 146 & 4.8 & 110 & 5.3 \\
\hline \multicolumn{7}{|l|}{ Chronic illness } \\
\hline No & 3,040 & 50.2 & $|, 42|$ & 46.9 & 872 & 41.9 \\
\hline Yes & 3,015 & 49.8 & 1,609 & 53.1 & 1,209 & 58.1 \\
\hline \multicolumn{7}{|l|}{ Nationality } \\
\hline German & 5,814 & 95.4 & 2,923 & 95.8 & 1,999 & 95.2 \\
\hline Other & 281 & 4.6 & 128 & 4.2 & 101 & 4.8 \\
\hline \multicolumn{7}{|l|}{ Reason for last physician visit } \\
\hline Preventive examination, immunization & 1,063 & 23.6 & 552 & 22.1 & 510 & 25.7 \\
\hline Chronic illness & 1,058 & 23.5 & 542 & 21.7 & 511 & 25.7 \\
\hline Current problem & 2,158 & 48.0 & $1,3 \mid 4$ & 52.5 & 842 & 42.3 \\
\hline Other & 218 & 4.9 & 93 & 3.7 & 125 & 6.3 \\
\hline
\end{tabular}

Notes: *Of all valid answers. Differences of sums to 6,105 (total), 3,056 (GPs) and 2,102 (SPs): no answer."Basic education" comprises eight or nine years of schooling; "intermediate" ten years; "higher" twelve or 13 years.

Abbreviations: GP, general practitioners; SP, specialists.Differences of sums to 6,I05 (total), 3,056 (GPs) and 2, 102 (SPs): no answer."Basic education”" comprises eight or nine years of schooling; "intermediate" ten years; "higher" twelve or 13 years. 
Table 3 Bivariate results for respondents who last consulted a general practitioner (GP) (n=3,056; weighted)

\begin{tabular}{|c|c|c|c|c|c|c|c|c|c|c|}
\hline \multirow{2}{*}{$\begin{array}{l}\text { Question on: } \\
\text { Answer }\end{array}$} & \multicolumn{2}{|c|}{$\begin{array}{l}\text { Main pro- } \\
\text { blem? }\end{array}$} & \multicolumn{2}{|c|}{$\begin{array}{l}\text { What to } \\
\text { do? }\end{array}$} & \multicolumn{2}{|c|}{$\begin{array}{l}\text { Why do } \\
\text { it? }\end{array}$} & \multicolumn{4}{|c|}{ Written materials? } \\
\hline & Yes & No & Yes & No & Yes & No & $\begin{array}{l}\text { Very } \\
\text { well }\end{array}$ & Well & $\begin{array}{l}\text { Not } \\
\text { well }\end{array}$ & $\begin{array}{l}\text { Not well at } \\
\text { all }\end{array}$ \\
\hline Total percentage & 93.9 & 6.1 & 95.4 & 4.6 & 98.8 & 1.2 & 22.6 & 64.1 & 12.0 & $\mathrm{I} .4$ \\
\hline Age (total) & 94.0 & 6.0 & 95.3 & 4.7 & 98.9 & I.I & 22.6 & 64.1 & 11.9 & 1.4 \\
\hline 18 to 34 years & 96.6 & 3.4 & 94.6 & 5.4 & 98.6 & 1.4 & 27.9 & 64.6 & 7.3 & 0.2 \\
\hline 35 to 49 years & 92.9 & 7.1 & 96.2 & 3.8 & 98.7 & 1.3 & 24.9 & 63.1 & 9.8 & 2.2 \\
\hline 50 to 64 years & 93.1 & 6.9 & 95.5 & 4.5 & 98.2 & 1.8 & 22.2 & 62.8 & 13.7 & 1.3 \\
\hline 65 years and above & 93.9 & 6.1 & 94.9 & 5.1 & 100.0 & 0.0 & 18.3 & 65.5 & 14.6 & 1.6 \\
\hline $\mathbf{n}$ & \multicolumn{2}{|c|}{$\mathrm{I}, \mathbf{4 7 8}$} & \multicolumn{2}{|l|}{1,289} & \multicolumn{2}{|l|}{784} & \multicolumn{4}{|l|}{$2,9 \mid 4$} \\
\hline Gender (total) & 94.0 & 6.0 & 95.3 & 4.7 & 98.9 & I.I & 22.5 & 64.1 & 12.0 & 1.4 \\
\hline Female & 93.8 & 6.2 & 95.5 & 4.5 & 99.0 & 1.0 & 23.7 & 64.0 & 11.1 & 1.2 \\
\hline Male & 94.2 & 5.8 & 95.1 & 4.9 & 98.7 & 1.3 & 21.3 & 64.2 & 13.0 & 1.5 \\
\hline $\mathbf{n}$ & \multicolumn{2}{|c|}{1,478} & \multicolumn{2}{|l|}{1,289} & \multicolumn{2}{|l|}{783} & \multicolumn{4}{|l|}{2,914} \\
\hline Educational status (total) & 94.1 & 5.9 & 95.3 & 4.7 & 98.7 & 1.3 & 22.5 & 64.3 & 12.0 & 1.2 \\
\hline Higher education & 94.2 & 5.8 & 93.8 & 6.2 & 98.4 & 1.6 & 36.9 & 58.1 & 4.6 & 0.5 \\
\hline Intermediate education & 92.9 & 7.1 & 95.7 & 4.3 & 98.1 & 1.9 & 21.3 & 65.6 & 12.2 & 0.9 \\
\hline Basic education & 95.5 & 4.5 & 96.5 & 3.5 & 99.6 & 0.4 & 11.7 & 68.3 & 17.8 & 2.1 \\
\hline $\mathbf{n}$ & \multicolumn{2}{|c|}{1,434} & \multicolumn{2}{|l|}{1,258} & \multicolumn{2}{|l|}{765} & \multicolumn{4}{|l|}{2,820} \\
\hline Occupational status (total) & 93.9 & 6.1 & 95.6 & 4.4 & 98.8 & 1.2 & 22.7 & 64.2 & 11.8 & 1.4 \\
\hline Full-time & 92.9 & 7.1 & 95.8 & 4.2 & 98.5 & 1.5 & 24.0 & 64.0 & 10.5 & 1.5 \\
\hline Part-time/temporary & 94.2 & 5.8 & 93.6 & 6.4 & 98.6 & $\mathrm{I} .4$ & 29.2 & 59.9 & 9.1 & 1.8 \\
\hline Unemployed & 100.0 & 0.0 & 100.0 & 0.0 & 100.0 & 0.0 & 11.5 & 78.8 & 7.7 & 1.9 \\
\hline Retired & 93.7 & 6.3 & 95.2 & 4.8 & 99.0 & 1.0 & 18.1 & 65.6 & 14.9 & 1.4 \\
\hline Training/volunteering/ & 97.2 & 2.8 & 100.0 & 0.0 & 100.0 & 0.0 & 32.8 & 59.0 & 8.2 & 0.0 \\
\hline school & 97.2 & 2.8 & 96.9 & 3.1 & 100.0 & 0.0 & 20.2 & 67.4 & 12.4 & 0.0 \\
\hline \multicolumn{11}{|l|}{ Parental leave/not- employed } \\
\hline $\mathbf{n}$ & \multicolumn{2}{|c|}{1,458} & \multicolumn{2}{|l|}{1,272} & \multicolumn{2}{|l|}{775} & \multicolumn{4}{|l|}{2,869} \\
\hline Type of health insurance (total) & 93.9 & 6.1 & 95.4 & 4.6 & 98.8 & 1.2 & 22.7 & 64.1 & 12.0 & 1.3 \\
\hline Private & 95.9 & 4.1 & 94.5 & 5.5 & 98.6 & 1.4 & 31.9 & 61.8 & 5.3 & 1.1 \\
\hline Statutory & 93.6 & 6.4 & 95.5 & 4.5 & 98.9 & 1.1 & 21.6 & 64.4 & 12.7 & 1.3 \\
\hline $\mathbf{n}$ & \multicolumn{2}{|c|}{1,469} & 1,275 & & 774 & & 2,883 & & & \\
\hline Self-reported health status (total) & 94.1 & 5.9 & 95.4 & 4.6 & 98.8 & 1.2 & 22.5 & 64.2 & 11.9 & 1.4 \\
\hline Excellent/very good & 95.5 & 4.5 & 93.8 & 6.2 & 97.7 & 2.3 & 30.2 & 59.0 & 9.8 & 1.0 \\
\hline Good & 94.2 & 5.8 & 96.3 & 3.7 & 99.5 & 0.5 & 18.9 & 67.3 & 12.1 & 1.7 \\
\hline Poor & 92.3 & 7.7 & 95.8 & 4.2 & 100.0 & 0.0 & 17.7 & 67.1 & 14.3 & 0.9 \\
\hline Very poor & 87.7 & 12.3 & 96.2 & 3.8 & 97.4 & 2.6 & 20.6 & 60.3 & 16.9 & 2.2 \\
\hline $\mathbf{n}$ & 1,468 & & 1,280 & & 781 & & 2,891 & & & \\
\hline Chronic illness (total) & 93.9 & 6.1 & 95.4 & 4.6 & 98.8 & 1.2 & 22.3 & 64.3 & 12.0 & 1.4 \\
\hline No & 94.3 & 5.7 & 95.8 & 4.2 & 99.0 & 1.0 & 22.0 & 66.3 & 10.5 & 1.2 \\
\hline Yes & 93.5 & 6.5 & 94.9 & 5.1 & 98.7 & 1.3 & 22.6 & 62.5 & 13.4 & 1.6 \\
\hline $\mathbf{n}$ & 1,466 & & 1,276 & & 779 & & 2,892 & & & \\
\hline
\end{tabular}


Table 3 (Continued).

\begin{tabular}{|c|c|c|c|c|c|c|c|c|c|c|}
\hline \multirow{2}{*}{$\begin{array}{l}\text { Question on: } \\
\text { Answer }\end{array}$} & \multicolumn{2}{|c|}{$\begin{array}{l}\text { Main pro- } \\
\text { blem? }\end{array}$} & \multicolumn{2}{|c|}{$\begin{array}{l}\text { What to } \\
\text { do? }\end{array}$} & \multicolumn{2}{|c|}{$\begin{array}{l}\text { Why do } \\
\text { it? }\end{array}$} & \multicolumn{4}{|c|}{ Written materials? } \\
\hline & Yes & No & Yes & No & Yes & No & $\begin{array}{l}\text { Very } \\
\text { well }\end{array}$ & Well & $\begin{array}{l}\text { Not } \\
\text { well }\end{array}$ & $\begin{array}{l}\text { Not well at } \\
\text { all }\end{array}$ \\
\hline Nationality (total) & 93.9 & 6.1 & 95.3 & 4.7 & 98.8 & 1.2 & 22.5 & 64.1 & 12.0 & 1.4 \\
\hline German & 93.8 & 6.2 & 95.4 & 4.6 & 99.1 & 0.9 & 22.7 & 64.3 & 11.6 & 1.4 \\
\hline Other & 95.7 & 4.3 & 93.7 & 6.3 & 95.0 & 5.0 & 19.5 & 59.3 & 20.3 & 0.8 \\
\hline $\mathbf{n}$ & \multicolumn{2}{|c|}{$\mathrm{I}, 475$} & \multicolumn{2}{|l|}{1,287} & \multicolumn{2}{|l|}{782} & \multicolumn{4}{|l|}{2,911} \\
\hline Reason for last physician visit & 93.8 & 6.2 & 95.5 & 4.5 & 99.1 & 0.9 & 23.5 & 63.2 & 12.1 & 1.3 \\
\hline (total) & 94.5 & 5.5 & 99.0 & 1.0 & 99.3 & 0.7 & 24.3 & 64.1 & 11.2 & 0.4 \\
\hline Preventive examination/immunization & 92.8 & 7.2 & 96.0 & 4.0 & 99.3 & 0.7 & 19.7 & 60.1 & 16.8 & 3.5 \\
\hline Chronic illness & 93.9 & 6.1 & 94.5 & 5.5 & 98.9 & 1.1 & 24.7 & 64.1 & 10.5 & 0.8 \\
\hline \multicolumn{11}{|l|}{ Current problem } \\
\hline$n$ & \multicolumn{2}{|c|}{1,425} & \multicolumn{2}{|c|}{1,254} & \multicolumn{2}{|l|}{763} & \multicolumn{4}{|l|}{2,306} \\
\hline
\end{tabular}

Notes: Differences of sums to 3,056: no answer. Gray background: statistically significant at $p \leq 0.05$.

The multivariable analysis confirmed these associations for patients with very poor self-reported health (OR: 3.17; CI: 1.32-7.61, reference group: excellent/very good health) and for those who saw the GP due to a current problem (OR: 6.54; CI: 1.70-25.12, reference group: preventive examination) (Table 5). It further revealed that being 35 to 49 years of age (OR: 2.33; CI: 1.06-5.14) was associated with limited understanding of one's health issue but with better comprehension of the treatment the GP recommended (OR: 0.39; CI: 0.17-0.88) compared to the reference group of 18- to 34-year-old patients (Table 5).

\section{Communication at the SP}

The bivariate outcomes for SP consultations showed that nearly one in five (19.4\%) of those who assessed their health as very poor reported not having understood their own health problem (vs $5.1 \%$ of those in excellent/very good health) (Table 4).

Taking all independent variables into account while adjusting for age and gender, this association remained: The OR for those suffering from very poor health to not understand their health problem was over five times higher than for the reference group (5.19; CI: 2.23-12.10) (Table 5). Moreover, patients aged 65 and above were more likely to not understand their own health issue (OR: 2.97; CI: 1.10-8.00, reference group: 18 to 34 years) (Table 5).

\section{Written health information}

Several socioeconomic variables were bivariately associated with patients' understanding of written health materials. Most notably, patients who last consulted the GP because of a chronic illness (20.3\%), with basic education (19.9\%) and those reporting poor health (19.1\%) answered that they did not understand written materials well or well at all (Table 3). After accounting for all independent variables in the model, the associations with basic education (OR: 4.20; CI: 2.76-6.39) and intermediate education (OR: 2.66; CI: 1.75-4.05, reference group: higher education), nationality other than German (OR: 3.78, CI: 2.12-6.74, vs German), statutory health insurance (OR: 2.26; CI: $1.17-4.35$, vs private) as well as higher age (50 to 64 years: OR: 2.09; CI: $1.29-3.41 ; 65$ years old and above: OR: 1.72; CI: $1.03-2.88$, reference group: 18 to 34 years-olds) were most pronounced (Table 5).

Of all patients who last consulted an SP, those with basic education (18.9\%), aged 65 years and above $(17.2 \%)$, retired $(16.8 \%)$ and with self-reported poor health $(16.2 \%)$ most often answered that they did not understand written information well or well at all (Table 4). Adjusted for all independent variables, basic education (OR: 4.63; CI: 2.84-7.54) as well as old age (65 years old and above: OR: 2.66, CI: 1.43-4.96) remained statistically significant (Table 5). 
Table 4 Bivariate results for respondents who last consulted a specialist (SP) ( $n=2,102$; weighted)

\begin{tabular}{|c|c|c|c|c|c|c|c|c|c|c|}
\hline \multirow{2}{*}{$\begin{array}{l}\text { Question: } \\
\text { Answer }\end{array}$} & \multicolumn{2}{|c|}{$\begin{array}{l}\text { Main pro- } \\
\text { blem? }\end{array}$} & \multicolumn{2}{|c|}{$\begin{array}{l}\text { What to } \\
\text { do? }\end{array}$} & \multicolumn{2}{|c|}{$\begin{array}{l}\text { Why do } \\
\text { it? }\end{array}$} & \multicolumn{4}{|c|}{ Written materials? } \\
\hline & Yes & No & Yes & No & Yes & No & $\begin{array}{l}\text { Very } \\
\text { well }\end{array}$ & Well & $\begin{array}{l}\text { Not } \\
\text { well }\end{array}$ & $\begin{array}{l}\text { Not well at } \\
\text { all }\end{array}$ \\
\hline Total percentage & 93.0 & 7.0 & 95.8 & 4.2 & 97.4 & 2.6 & 25.5 & 64.2 & 9.3 & 1.0 \\
\hline Age (total) & 93.0 & 7.0 & 95.8 & 4.2 & 97.5 & 2.5 & 25.5 & 64.2 & 9.3 & 1.0 \\
\hline 18 to 34 years & 95.9 & 4.1 & 95.4 & 4.6 & 97.0 & 3.0 & 31.9 & 63.6 & 4.5 & 0.0 \\
\hline 35 to 49 years & 93.4 & 6.6 & 96.1 & 3.9 & 99.4 & 0.6 & 29.1 & 62.0 & 8.0 & 0.8 \\
\hline 50 to 64 years & 92.2 & 7.8 & 95.8 & 4.2 & 96.6 & 3.4 & 24.5 & 66.5 & 8.9 & 0.2 \\
\hline 65 years and above & 91.4 & 8.6 & 96.0 & 4.0 & 96.5 & 3.5 & 18.5 & 64.3 & 14.4 & 2.8 \\
\hline $\mathbf{n}$ & \multicolumn{2}{|l|}{1,207} & \multicolumn{2}{|l|}{936} & \multicolumn{2}{|l|}{629} & \multicolumn{4}{|l|}{$\mathbf{I , 9 9 |}$} \\
\hline Gender (total) & 93.0 & 7.0 & 95.8 & 4.2 & 97.3 & 2.7 & 25.5 & 64.2 & 9.3 & 1.0 \\
\hline Female & 92.7 & 7.3 & 94.4 & 5.6 & 97.3 & 2.7 & 27.5 & 63.7 & 7.9 & 1.0 \\
\hline Male & 93.3 & 6.7 & 97.5 & 2.5 & 97.3 & 2.7 & 22.7 & 64.9 & 11.3 & I.I \\
\hline $\mathbf{n}$ & \multicolumn{2}{|l|}{1,208} & \multicolumn{2}{|l|}{936} & \multicolumn{2}{|l|}{631} & \multicolumn{4}{|l|}{ I,992 } \\
\hline Educational status (total) & 92.9 & 7.1 & 95.9 & 4.1 & 97.4 & 2.6 & 25.6 & 64.1 & 9.2 & I.I \\
\hline Higher education & 93.7 & 6.3 & 96.2 & 3.8 & 97.6 & 2.4 & 36.6 & 59.6 & 3.6 & 0.3 \\
\hline Intermediate education & 93.5 & 6.5 & 94.8 & 5.2 & 96.7 & 3.3 & 24.7 & 64.6 & 10.1 & 0.6 \\
\hline Basic education & 91.2 & 8.8 & 97.1 & 2.9 & 98.2 & 1.8 & II.I & 70.0 & 16.0 & 2.9 \\
\hline $\mathbf{n}$ & \multicolumn{2}{|l|}{1,169} & \multicolumn{2}{|l|}{912} & \multicolumn{2}{|l|}{616} & \multicolumn{4}{|l|}{1,940} \\
\hline Occupational status (total) & 92.9 & 7.1 & 96.0 & 4.0 & 97.3 & 2.7 & 25.3 & 64.3 & 9.4 & 1.0 \\
\hline Full-time & 93.5 & 6.5 & 97.5 & 2.5 & 96.9 & 3.1 & 29.2 & 63.6 & 6.5 & 0.7 \\
\hline Part-time/temporary & 95.6 & 4.4 & 95.8 & 4.2 & 98.0 & 2.0 & 26.0 & 65.1 & 8.9 & 0.0 \\
\hline Unemployed & 100.0 & 0.0 & 83.3 & 16.7 & 100.0 & 0.0 & 23.5 & 64.7 & 11.8 & 0.0 \\
\hline Retired & 91.1 & 8.9 & 95.5 & 4.5 & 96.5 & 3.5 & 18.3 & 64.9 & 14.4 & 2.4 \\
\hline Training/volunteering/ & 93.5 & 6.5 & 90.2 & 9.8 & 100.0 & 0.0 & 34.4 & 63.5 & 2.1 & 0.0 \\
\hline school & 89.4 & 10.6 & 96.6 & 3.4 & 100.0 & 0.0 & 28.1 & 64.1 & 7.8 & 0.0 \\
\hline Parental leave/not- employed & & & & & & & & & & \\
\hline $\mathbf{n}$ & \multicolumn{2}{|c|}{ I, I 88} & \multicolumn{2}{|l|}{924} & \multicolumn{2}{|l|}{625} & \multicolumn{4}{|l|}{1,962} \\
\hline Type of health insurance (total) & 93.0 & 7.0 & 95.8 & 4.2 & 97.3 & 2.7 & 25.3 & 64.3 & 9.4 & 1.0 \\
\hline Private & 94.7 & 5.3 & 97.9 & 2.1 & 97.9 & 2.1 & 32.6 & 61.1 & 5.7 & 0.6 \\
\hline Statutory & 92.7 & 7.3 & 95.4 & 4.6 & 97.2 & 2.8 & 23.9 & 64.9 & 10.1 & 1.1 \\
\hline $\mathbf{n}$ & \multicolumn{2}{|c|}{1,200} & \multicolumn{2}{|l|}{930} & 626 & & 1,975 & & & \\
\hline Self-reported health status (total) & 93.1 & 6.9 & 96.1 & 3.9 & 97.5 & 2.5 & 25.4 & 64.3 & 9.3 & I.I \\
\hline Excellent/very good & 94.9 & 5.1 & 96.4 & 3.6 & 99.0 & 1.0 & 34.8 & 58.7 & 5.9 & 0.6 \\
\hline Good & 93.6 & 6.4 & 95.6 & 4.4 & 97.7 & 2.3 & 20.7 & 68.8 & 9.9 & 0.6 \\
\hline Poor & 92.7 & 7.3 & 96.4 & 3.6 & 95.6 & 4.4 & 19.3 & 64.4 & 13.7 & 2.5 \\
\hline Very poor & 80.6 & 19.4 & 97.6 & 2.4 & 93.3 & 6.7 & 24.0 & 64.0 & 10.0 & 2.0 \\
\hline $\mathbf{n}$ & 1,203 & & 932 & & 628 & & I,97I & & & \\
\hline Chronic illness (total) & 93.0 & 7.0 & 96.1 & 3.9 & 97.5 & 2.5 & 25.7 & 64.0 & 9.3 & 1.0 \\
\hline No & 94.6 & 5.4 & 95.7 & 4.3 & 97.1 & 2.9 & 27.3 & 63.9 & 8.3 & 0.5 \\
\hline Yes & 91.7 & 8.3 & 96.4 & 3.6 & 97.7 & 2.3 & 24.5 & 64.0 & 10.1 & 1.4 \\
\hline $\mathbf{n}$ & 1,198 & & 929 & & 628 & & 1,970 & & & \\
\hline
\end{tabular}


Table 4 (Continued).

\begin{tabular}{|c|c|c|c|c|c|c|c|c|c|c|}
\hline \multirow{2}{*}{$\begin{array}{l}\text { Question: } \\
\text { Answer }\end{array}$} & \multicolumn{2}{|c|}{$\begin{array}{l}\text { Main pro- } \\
\text { blem? }\end{array}$} & \multicolumn{2}{|c|}{$\begin{array}{l}\text { What to } \\
\text { do? }\end{array}$} & \multicolumn{2}{|c|}{$\begin{array}{l}\text { Why do } \\
\text { it? }\end{array}$} & \multicolumn{4}{|c|}{ Written materials? } \\
\hline & Yes & No & Yes & No & Yes & No & $\begin{array}{l}\text { Very } \\
\text { well }\end{array}$ & Well & $\begin{array}{l}\text { Not } \\
\text { well }\end{array}$ & $\begin{array}{l}\text { Not well at } \\
\text { all }\end{array}$ \\
\hline Nationality (total) & 93.0 & 7.0 & 95.8 & 4.2 & 97.3 & 2.7 & 25.5 & 64.2 & 9.3 & 1.0 \\
\hline German & 92.8 & 7.2 & 95.8 & 4.2 & 97.4 & 2.6 & 25.6 & 64.0 & 9.4 & I.I \\
\hline Other & 95.8 & 4.2 & 96.5 & 3.5 & 95.5 & 4.5 & 24.0 & 68.8 & 7.3 & 0.0 \\
\hline $\mathbf{n}$ & \multicolumn{2}{|c|}{1,206} & \multicolumn{2}{|l|}{934} & \multicolumn{2}{|l|}{629} & \multicolumn{4}{|l|}{1,989} \\
\hline Reason for last physician visit & 93.0 & 7.0 & 96.0 & 4.0 & 97.1 & 2.9 & 24.9 & 64.9 & 9.2 & 1.0 \\
\hline (total) & 95.6 & 4.4 & 95.5 & 4.5 & 98.2 & 1.8 & 24.7 & 66.6 & 7.7 & 1.0 \\
\hline Preventive examination/immunization & 90.6 & 9.4 & 94.7 & 5.3 & 95.5 & 4.5 & 25.8 & 61.1 & 11.8 & 1.3 \\
\hline Chronic illness & 93.1 & 6.9 & 96.8 & 3.2 & 97.6 & 2.4 & 24.5 & 66.2 & 8.6 & 0.8 \\
\hline \multicolumn{11}{|l|}{ Current problem } \\
\hline$n$ & \multicolumn{2}{|c|}{ I, 126} & \multicolumn{2}{|l|}{881} & \multicolumn{2}{|l|}{596} & \multicolumn{4}{|l|}{1,763} \\
\hline
\end{tabular}

Notes: Differences of sums to 2,102: no answer. Gray background: statistically significant at $p \leq 0.05$.

\section{Discussion, implications and limitations \\ Discussion \\ Oral communication}

This study explored whether patients understand the health information they are likely to receive in case of illness. The principal finding is that the large majority of patients in outpatient care in Germany report that they understand the GPs' and SPs' explanations on their main health problem, what to do about it and why to do this. Marginal differences exist between GPs and SPs. This suggests that from a patient perspective, the level of understanding in patient-physician communication in Germany is high.

Yet, the results reveal that a share of patients does not understand the GPs' and SPs' explanations, especially those regarding the main health problem. Considering population subgroups, the understanding of oral health information differs between age groups, with older patients (65 years old above) reporting more often failing to understand explanations about their health problem. Further, patients who rate their health as very poor or report that they have a current health problem appear to have more difficulties understanding their physician. This indicates that personal health issues might often remain ambiguous for patients who are most in need of medical care.

A recent study on patients' understanding of GPs and SPs in Germany notes that $42 \%$ of all patients report that they fail to understand their GP's explanations and 48\% report that they do not understand their SP's explanations. $^{28}$ The respective survey, however, used a single question to ask patients whether they failed to fully understand their physician's explanations in the previous 12 months. $^{28}$ Our study provides more specific information on patients' understanding as we differentiated according to the AM3 questions. While existing international studies on AM3 focus on the intervention's effectiveness in increasing patient empowerment and satisfaction in outpatient care, ${ }^{25,26,29}$ none of these have examined patients' understanding of oral communication.

The associations between HL and socioeconomic variables have been explored in other studies, ${ }^{11,12,28}$ indicating that people 65 years old and above can be rated as having lower HL levels ${ }^{11,12,30}$ and find it more often difficult to generally understand health information. ${ }^{28}$ Yet, the associations between socioeconomic variables and the understanding of physicians' explanations have not been explicitly investigated before.

\section{Written communication}

Our analysis also shows that written health information is not well understood by more than one in ten patients, indicating that patients in Germany have more difficulties with understanding textual than oral health information.

Written health information seems to be insufficiently tailored to individuals aged 50 years and above as well as to those with intermediate and basic education. This 


\begin{tabular}{|c|c|c|c|c|c|c|c|c|c|}
\hline g & 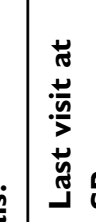 & is & 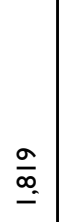 & ô তิ & 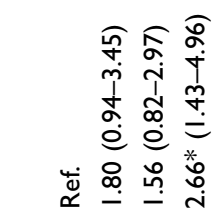 & & 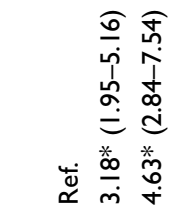 & & \\
\hline 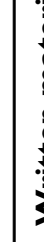 & 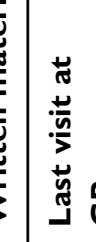 & i & $\frac{2}{i}$ & ô তิ & 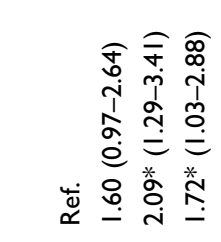 & 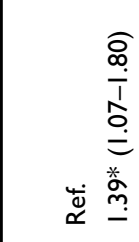 & 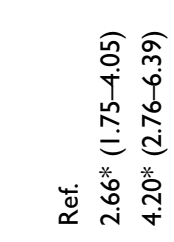 & & 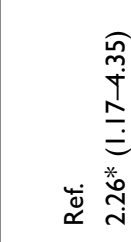 \\
\hline & 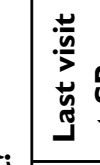 & 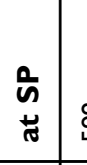 & $\begin{array}{c}\infty \\
\stackrel{\infty}{0} \\
\end{array}$ & ô $\widehat{\bar{Q}}$ & & & & & \\
\hline 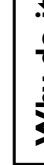 & 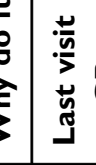 & $\begin{array}{lll} & \\
0 & \\
\end{array}$ & $\stackrel{0}{n}$ & of $\overline{\mathrm{v}}$ & & & & & \\
\hline & 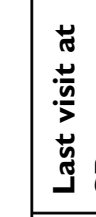 & & $\stackrel{\swarrow}{\infty}$ & ô ত్రิ & & & & & \\
\hline ? & 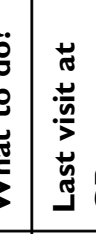 & ¿ & $\stackrel{\tilde{\infty}}{=}$ & ô তิ & 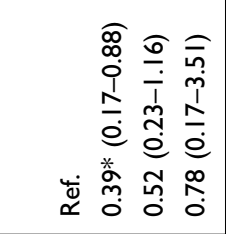 & & & 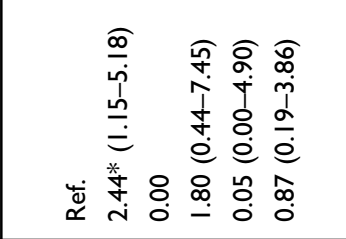 & \\
\hline & 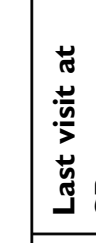 & is & $\stackrel{\widetilde{\sigma}}{=}$ & oิ త్రิ & 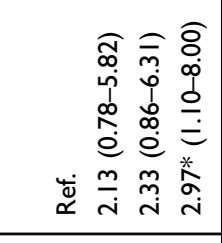 & & & & \\
\hline & 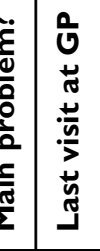 & & $\stackrel{\substack{0 \\
\hdashline}}{=}$ & ơ তิ & 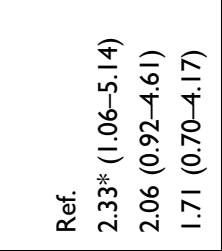 & & & & \\
\hline & כ. & & 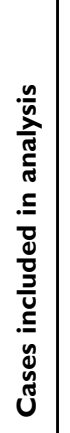 & & 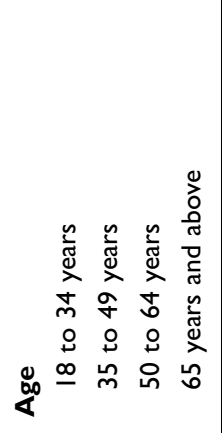 & 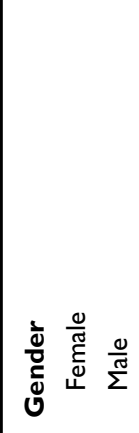 & 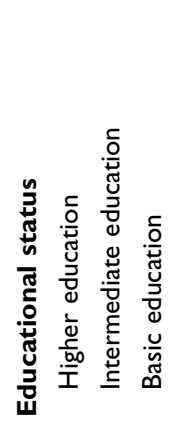 & 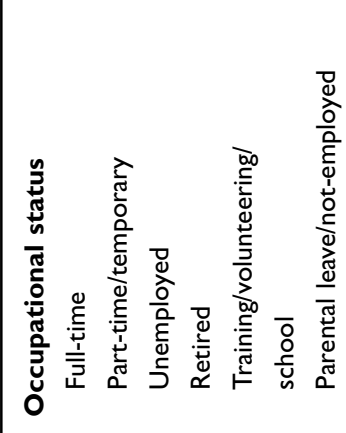 & 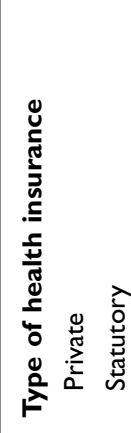 \\
\hline
\end{tabular}




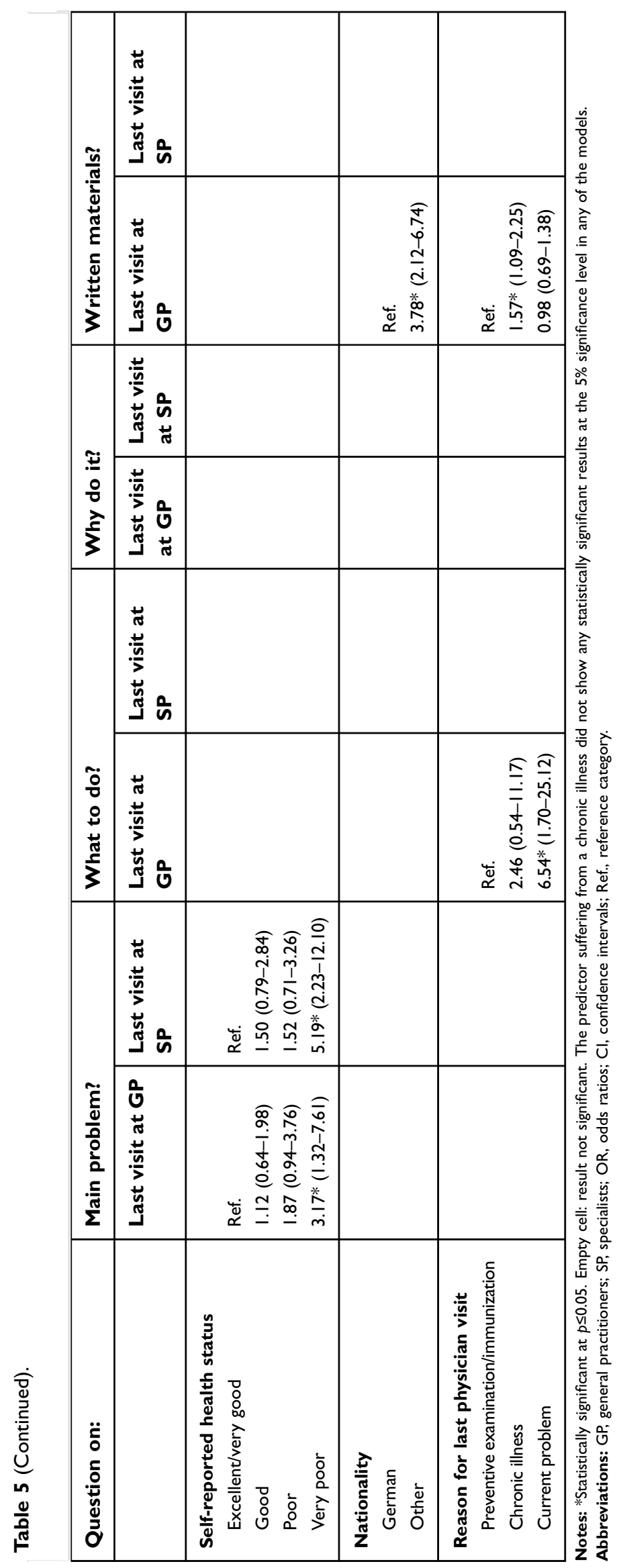


suggests that in order to increase these populations' understanding of their own health, textual materials might need to be simplified and adapted to their communication capacities, for example by avoiding medicalized language but using instructional graphics and pictograms instead- ${ }^{33}$

Similarly, the study by Schaeffer et al cited above found that people with basic education are more likely to experience difficulties in understanding health information in general than those with intermediate and higher education. $^{28}$ Thus, our study underlines the associations between low educational levels and problematic HL that have been identified previously. ${ }^{11,12,30}$

\section{Implications}

Adding to studies that show that patients' old age and selfreported poor health are correlated with longer waiting times for physician appointments ${ }^{23}$ and with worse patients' assessment of health system responsiveness, ${ }^{34}$ our study's results suggest that certain patient groups are at higher risks than others to experience non-medical difficulties in the outpatient care process in Germany.

According to Lambert et al "ensuring patient knowledge (...) of all important aspects of their condition and treatment" ${ }^{\prime 35}$ is crucial to improving health care delivery and patient health outcomes. ${ }^{35}$ Thus, efforts to enhance oral communication between physicians and patients 65 years of age and above, with very poor self-reported health and a current health problem can be assumed to improve the quality of outpatient care for a critical patient population. As an initiating step, measures could include integrating HL strategies and communication tools into medical training curricula. $^{18,21,36}$ These actions might contribute to raising physicians' general awareness of problematic HL among patients, ${ }^{35,37,38}$ as well as to practicing techniques to facilitate effective interpersonal communication with patients of the identified risk-groups. Tailoring textual health materials to the competences of older patients and those with lower education may further facilitate these groups' understanding of health issues and foster patient empowerment. ${ }^{33}$ This may respect the strong association between general literacy and health literacy. ${ }^{10}$ As one in seven adults - particularly of older age and with lower educational level - shows below basic general literacy skills in the $\mathrm{US}^{39}$ and Germany ${ }^{40}$ these individuals undergo hardships once they face routine health and health-care tasks which often require more literacy skills than those of everyday life. ${ }^{10}$

A study by Wolf et al suggests that patients for whom written health materials are inadequate tend to rely exclusively on the physicians' explanations, ${ }^{41}$ whereas high quality and easy-to-understand written information can support the patient-physician interaction. ${ }^{42}$ This interdependence between oral and written communication might also exist in Germany, implying that in order to effectively communicate health information, oral and written dissemination styles need to be combined. ${ }^{31,32,43}$

\section{Limitations}

Limitations of our results include that a complete analysis of the patient-physician communication in Germany would need to scrutinize inpatient care as well. Deficits in understanding physicians in hospitals and other care institutions might be more pronounced than in outpatient care as patients are free to consult any GP and SP in Germany - and might choose a physician whom they understand well -, whereas hospital-based physicians are more often consulted due to acute, unanticipated health issues which means that patients are less likely to select the hospital physician.

Also, our survey asked for general understanding of written health information. Differences between the provider of the information (for example hospitals, sickness funds, health care authorities) or the type of material (for example medical package insert, medical journal, leaflet) were not accounted for.

Further, our analysis was based exclusively on patients' subjective answers, not taking into account any actual assessment of whether the patients did understand the provided information. Study participants' recall error and communicative limitations may have additionally distorted their evaluation of the last physician consultation. ${ }^{23,44}$

Similar to Schaeffer et al $^{28}$ interviewing only Germanspeakers excluded persons with limited German skills who may have greater difficulties understanding their Germanspeaking physicians, which could have positively skewed our results. While the final sample was weighted for sex, age and education according to their nominal distribution across Germany's population, there are no official numbers for the German-speaking population 18 years old and above from which the sample had been drawn, leaving our sample inexact to some extent.

In addition, as this survey was conducted via landline only, individuals without a phone connection were excluded. This may have meant that certain patient sub groups were unable to participate in the survey, notably young adults who do not live at their parents' house (for example university students) as well as people of older age 
who do not reside in private households (for example, those living in care facilities).

\section{Conclusion}

This paper is the first to present results on patients' understanding of GPs' and SPs' explanations operationalized using the AM3 questions and of written health information in Germany.

While the findings suggest that patients' understanding of oral communication during outpatient consultations and written health materials is satisfactory, certain patient sub groups report deficits regarding patient-physician communication and comprehensibility of written health materials. Meeting the communication needs of, and improving communication with, elderly patients, those with basic education and (very) poor self-reported health should be a priority in patient-physician interactions. In combination with interventions that increase HL training for health professionals, reduce system barriers and combine oral and written health communication this may lead to better patients' understanding and help to improve outpatient care quality in Germany.

\section{Data availability}

The data that this study has drawn on may be provided upon request.

\section{Ethics}

This research was exempt from ethics approval by the ethics committee of the Charité - Universitätsmedizin Berlin as it involved the use of records that only contain anonymized, purely observational, noninvasive and noninteractive data about study participants, and did not involve any (medical) treatment at any point.

\section{Acknowledgments}

We acknowledge support for the open access publication from the German Research Foundation (DFG) and the Open Access Publication Fund of Charité Universitätsmedizin Berlin. No financial assistance was received to conduct the study described in the manuscript, or used to assist with the preparation of the manuscript.

\section{Disclosure}

Heide Weishaar reports being a research fellow on a project aimed at developing a national action plan for health literacy in Germany whilst writing the manuscript. The authors report no other conflicts of interest in this work.

\section{References}

1. Rudd RE. Health Literacy: insights and Issues. Stud Health Technol Inform. 2017a;240:60-78.

2. Pelikan JM, Dietscher C. Warum sollten und wie können Krankenhäuser ihre organisationale Gesundheitskompetenz verbessern? Bundesgesundheitsbl. 2015;58:989-995. doi:10.1007/s00103015-2206-6

3. Rudd RE. Health literacy developments, corrections, and emerging themes. In: Schaeffer D, Pelikan JM, editors. Health Literacy, Forschungsstand und Perspektiven. 1st ed. Bern: Hogrefe; 2017b:19-32.

4. Schaeffer D, Pelikan JM. Health Literacy: begriff, Konzept, Relevanz. In: Schaeffer D, Pelikan JM, editors. Health Literacy, Forschungsstand und Perspektiven. 1st ed. Bern: Hogrefe; 2017a:11-18.

5. Lee SY, Arozullah AM, Cho YI. Health literacy, social support, and health: a research agenda. Soc Sci Med. 2004;58:1309-1321.

6. World Health Organization. Shanghai Declaration on Promoting Health in the 2030 Agenda for Sustainable Development. Shanghai: WHO; 2016. Available from: http://www.who.int/healthpromotion/ conferences/9gchp/shanghai-declaration/en/. Accessed March 1, 2019.

7. Sørensen K, Van Den Broucke S, Fullam J, et al. for (HLS-EU) Consortium Health Literacy Project European. Health literacy and public health: A systematic review and integration of definitions and models. BMC Public Health. 2012;12:80. doi:10.1186/1471-2458-1280

8. Dietscher C, Pelikan JM. Health-literate hospitals and healthcare organizations - results from an Austrian feasibility study on the selfassessment of organizational health literacy in hospitals. In: Schaeffer D, Pelikan JM, editors. Health Literacy, Forschungsstand und Perspektiven. 1st ed. Bern: Hogrefe; 2017:303-314.

9. Berkman ND, Sheridan SL, Donahue KE, Halpern DJ, Crotty K. Low health literacy and health outcomes: an updated systematic review. Ann Intern Med. 2011;155:97-107. doi:10.7326/0003-4819-1552-201107190-00005

10. Institute of Medicine, Board on Neuroscience and Behavioral Health, Committee on Health Literacy. Health Literacy: A Prescription to End Confusion. Washington, DC: The National Academies Press; 2004.

11. Kutner M, Greenberg E, Jin Y, Paulsen C. The Health Literacy of America's Adults: Results from the 2003 National Assessment of Adult Literacy (NCES 2006-483). Washington, DC: U.S. Department of Education, National Center For Education Statistics; 2006.

12. The HLS-EU Consortium. Comparative Report on Health Literacy in Eight EU Member States (Second Extended and Revised Version). n/ a; 2014. Available from: https://cdn1.sph.harvard.edu/wp-content /uploads/sites/135/2015/09/neu_rev_hls-eu_report_2015_05_13_lit. pdf. Accessed March 1, 2019.

13. Schaeffer D, Berens EM, Vogt D. Health literacy in the German population - results of a representative survey. Dtsch Ärztebl Int. 2017;114:53-60.

14. Pelikan JM, Ganahl K. Die europäische GesundheitskompetenzStudie: konzept, Instrument und ausgewählte Ergebnisse. In: Schaeffer D, Pelikan JM, editors. Health Literacy, Forschungsstand und Perspektiven. 1st ed. Bern: Hogrefe; 2017:93-126.

15. Quenzel G, Schaeffer D, Messer M, Vogt D. Health Literacy und Gesundheitsverhalten vulnerabler Bevölkerungsgruppen. In: Schaeffer D, Pelikan JM, editors. Health Literacy, Forschungsstand und Perspektiven. 1st ed. Bern: Hogrefe; 2017:157-174.

16. World Health Organization. Health Literacy. The Solid Facts. Copenhagen. WHO; 2013. Available from: http://www.euro.who. int/_data/assets/pdf_file/0008/190655/e96854.pdf. Accessed March 1,2019 . 
17. Schaeffer D, Pelikan JM. Health Literacy - künftige Herausforderungen. In: Schaeffer D, Pelikan JM, editors. Health Literacy, Forschungsstand und Perspektiven. 1st ed. Bern: Hogrefe; 2017:315-320.

18. Dietscher C, Lorenc J, Pelikan JM. Selbstbewertung organisationaler Gesundheitskompetenz. Machbarkeitsstudie zum Selbstbewertungsinstrument nach dem Wiener Konzept Gesundheitskompetenter Krankenbehandlungsorganisationen (WKGKKO-I). Wien: LBIHPR; 2015.

19. Hernandez LM. Measures of Health Literacy: Workshop Summary. Washington, DC: The National Academies Press; 2009.

20. Institute of Medicine, USA. Ten Attributes of Health Literate Health Care Organizations. Discussion paper. Washington, DC: Institute of Medicine; 2012. Available from: https://nam.edu/wp-content/uploads/ 2015/06/BPH_Ten_HLit_Attributes.pdf. Accessed March 1, 2019.

21. Coleman C, Hudson S, Pederson B. Prioritized health literacy and clear communication practices for health care professionals. Hlrp. 2017;1:e91-99.

22. Tsai TI, Yu WR, Lee SY. Is health literacy associated with greater medical care trust? Int J Qual Health Care. 2018;30:514-519. doi:10.1093/intqhe/mzy043

23. Tille F, Gibis B, Balke K, Kuhlmey A, Schnitzer S. Sociodemographic and health-related determinants of health care utilisation and access to primary and specialist care: results of a nationwide population survey in Germany (2006-2016). Z Evid Fortbild Qual Gesundhwes. 2017;126:52-65. doi:10.1016/j.zefq.2017.07.012

24. Forschungsgruppe Wahlen Telefonfeld GmbH. Versichertenbefragung der Kassenärztlichen Bundesvereinigung. Mannheim: Forschungsgruppe Wahlen; 2017. Available from: http:// www.kbv.de/media/sp/Berichtband_KBV_Versichertenbefragung 2017.pdf. Accessed March 1, 2019.

25. Groene RO, Bolíbar I, Brotons C. Impact, barriers and facilitators of the 'Ask Me 3' patient communication intervention in a primary care center in Barcelona, Spain: a mixed-methods analysis. Int J Pers Cent Med. 2012;2:853-861.

26. Galliher JM, Post DM, Weiss BD, et al. Patients' question-asking behavior during primary care visits: a report from the AAFP National Research Network. Ann Fam Med. 2010;8:151-159. doi:10.1370/afm.1055

27. Institute for Healthcare Improvement. Ask Me 3: Good Questions for Your Good Health. Boston: Institute for Healthcare Improvement; 2018. Available from: http://www.npsf.org/page/askme3. Accessed March 1, 2019.

28. Schaeffer D, Vogt D, Berens EM, Hurrelmann K. Gesundheitskompetenz der Bevölkerung in Deutschland Ergebnisbericht. Bielefeld: Universität Bielefeld; 2016.

29. Michalopoulou G, Falzarano P, Arfken C, Rosenberg D. Implementing Ask $\mathrm{Me} 3^{\mathrm{TM}}$ to Improve African American patient satisfaction and perceptions of physician cultural competency. J Cult Divers. 2010;17:62-67.

30. Berens EM, Vogt D, Messer M, Hurrelmann K, Schaeffer D. Health literacy among different age groups in Germany: results of a cross-sectional survey. BMC Public Health. 2016;16:1151. doi:10.1186/s12889-016-3810-6
31. Parker R. Health literacy: a challenge for American patients and their health care providers. Health Promot Int. 2000;15:277-283. doi:10.1093/heapro/15.4.277

32. Davis TC, Williams MV, Marin E, Parker RM, Glass J. Health literacy and cancer communication. $C A$ Cancer $J$ Clin. 2002;52:134-149.

33. Yin HS, Dreyer PB, van Schaick L, Foltin GL, Dinglas C, Mendelsohn AL. Randomized controlled trial of a pictogram-based intervention to reduce liquid medication dosing errors and improve adherence among caregivers of young children. Arch Pediatr Adolesc Med. 2008;162:814-822. doi:10.1001/archpedi.162.9.814

34. Tille F, Röttger J, Gibis B, Busse R, Kuhlmey A, Schnitzer S. Patients' perceptions of health system responsiveness in ambulatory care in Germany. Patient Educ Couns. 2019;102:162-171. doi:10.1016/j.pec.2018.08.020

35. Lambert M, Luke J, Downey B, et al. Health literacy: health professionals' understandings and their perceptions of barriers that indigenous patients encounter. BMC Health Serv Res. 2014;29:614. doi:10.1186/s12913-014-0614-1

36. Schwartzberg JG, Cowett A, van Geest J, Wolf MS. Communication techniques for patients with low health literacy: a survey of physicians, nurses, and pharmacists. Am J Health Behav. 2007;31:96-104. doi:10.5993/AJHB.31.s1.12

37. Schillinger D, Piette J, Grumbach K, et al. Closing the loop: physician communication with diabetic patients who have low health literacy. Arch Intern Med. 2003;163:83-90. doi:10.1001/ archinte.163.1.83

38. Bass PF, Wilson JF, Griffith CH, Barnett DR. Residents' ability to identify patients with poor literacy skills. Acad Med. 2002;77:1039-1041.

39. Kutner M, Greenberg E, Baer J. A First Look at the Literacy of America'S Adults in the 21st Century (NCES 2006-470). Washington, DC: U.S. Government Printing Office; 2005.

40. Universität Hamburg. Leo. Level-One Studie. Hamburg: Universität Hamburg; 2017. Available from: http://blogs.epb.uni-hamburg.de/leo/ ?cat=51745. Accessed March 1, 2019.

41. Wolf MS, Davis TC, Cross JT, Marin E, Green K, Bennett CL. Health literacy and patient knowledge in a Southern US HIV clinic. Int J STD AIDS. 2004;15:747-752. doi:10.1258/0956462042395131

42. Jacobson TA, Thomas DM, Morton FJ, Offut G, Shevlin J, Ray S. Use of a low-literacy patient education tool to enhance pneumococcal vaccination rates. A randomized controlled trial. JAMA. 1999;282:646-650. doi:10.1001/jama.282.7.646

43. Davis TC, Fredrickson DD, Arnold C, Murphy PW, Herbst M, Bocchini JA. A polio immunization pamphlet with increased appeal and simplified language does not improve comprehension to an acceptable level. Patient Educ Couns. 1998;33:25-37. doi:10.1016/ S0738-3991(97)00053-0

44. Huang YJ, Chen CT, Lin GH, et al. Evaluating the European health literacy survey questionnaire in patients with stroke: a latent trait analysis using rasch modeling. Patient. 2018;11:83-96.

\section{Publish your work in this journal}

Patient Preference and Adherence is an international, peer-reviewed, open access journal that focusing on the growing importance of patient preference and adherence throughout the therapeutic continuum. Patient satisfaction, acceptability, quality of life, compliance, persistence and their role in developing new therapeutic modalities and compounds to optimize clinical outcomes for existing disease states are major areas of interest for the journal. This journal has been accepted for indexing on PubMed Central. The manuscript management system is completely online and includes a very quick and fair peer-review system, which is all easy to use. Visit http:// www.dovepress.com/testimonials.php to read real quotes from published authors. 